\title{
IMPACTO DE LA MOTIVACIÓN EN LA PRODUCTIVIDAD EMPRESARIAL: CASO GERENCIA DE INFRAESTRUCTURA EN TELEFÓNICA DEL PERÚ
}

IMPACT ON MOTIVATION CORPORATE PRODUCTIVITY: CASE MANAGEMENT INFRASTRUCTURE TELEPHONE OF PERU.

\author{
Pedro L. Tito Huamaní \\ pedrotito18@hotmail.com \\ Pepe Acuña Oré ${ }^{\text {**** }}$ \\ pepe.acuna@telefonica.com
}

[RECEPCIÓN: MARZO DE 2015 / CONFORMIDAD: ABRIL DE 2015]

\section{RESUMEN}

Para el sector empresarial en general y peruano en particular, se hace cada vez más necesario tener una plantilla competitiva y motivada; pero esto no es fácil debido -entre otros aspectos.- a la presión de los medios con noticias relacionadas a la desaceleración de la economía, a la inseguridad y la corrupción, aspectos que distraen y hasta desmoralizan a los trabajadores. Además, vivimos en un mundo globalizado donde prima el aspecto competitivo, especialmente en el sector tecnológico y de telecomunicaciones, donde opera la empresa objeto del presente estudio.

La investigación se ha realizado en la Gerencia de Infraestructura de Telefónica del Perú, con la intención de determinar cómo la motivación contribuye en la productividad de los trabajadores, cuáles son los factores de mayor impacto y cuáles son los beneficios que el área obtiene. Se presentan algunos conceptos a modo de marco teórico, se desarrollan las hipótesis, se presenta la metodología empleada y se analizan los resultados; se adjuntan las conclusiones y recomendaciones pertinentes.

Palabras clave: Motivación, productividad.

\begin{abstract}
For the business sector in general and Peruvian in particular is becoming increasingly necessary to have a competitive and motivated workforce; but this is not easy due to pressure from the media related to the slowing economy, insecurity and corruption issues that distract and to demoralize workers. Also, we live in a globalized world where raw competitive aspect, especially in the technology and telecommunications sector, where the company object of this study is positioned.
\end{abstract}

\footnotetext{
* Doctor en Ciencias Administrativas (UNMSM), Magíster en Gestión Empresarial y Licenciado en Administración (UNMSM). Director del Instituto de Investigación de la Facultad de Ciencias Administrativas (FCA). Docente Asociado de la Facultad de Ciencias AdministrativasUNMSM. Docente de Posgrado de la UNMSM y docente invitado en las diferentes universidades del país y extranjero. Miembro del Directorio de Investigadores del CONCYTEC-Perú.

** Magíster en Administración con mención en Gestión Empresarial, Ing. Electrónico UNMSM. Docente de Posgrado de la FCA, UNMSM.
} 
The research has been done in the Infrastructure Management of Telefonica del Perú, with the intention of determining the motivation contributes to the productivity of workers, what are the factors of greatest impact and what are the benefits that the area gets. Some concepts as a theoretical framework presented, hypotheses are developed, the methodology is presented and the results are analyzed; accompanying the conclusions and recommendations.

Keywords: Motivation, productivity.

\section{INTRODUCCIÓN}

La motivación como proceso que impulsa a las personas a actuar de una determinada manera, es un aspecto fundamental en el ámbito empresarial. Las empresas buscan en sus cuadros a personas motivadas y entusiasmadas con su trabajo que les permitan alcanzar sus objetivos a través de un elevado estándar de desempeño. Por ello, motivar a las personas se constituye en un desafío para las organizaciones y sus líderes quienes buscan que sus colaboradores se sientan confiados y comprometidos con alcanzar los objetivos propuestos.

La influencia de la motivación en el desempeño de las personas es esencial para alcanzar determinados objetivos establecidos por las organizaciones. Por esta razón las empresas necesitan motivar constantemente a sus trabajadores y generar un sentimiento de pertenencia y compromiso.

En la presente investigación, se busca determinar cómo contribuye la motivación a la productividad de la Gerencia de Infraestructura de Telefónica del Perú, cuáles son los factores de motivación que tienen mayor impacto en los trabajadores y cuáles son los beneficios que obtiene el área de Infraestructura. La investigación se justifica en la práctica por la necesidad de Telefónica del Perú por conocer a sus trabajadores y saber qué es lo que genera en ellos una mayor motivación de trabajo y un mayor compromiso con la empresa.

\section{FICHA TÉCNICA}

El presente trabajo de investigación se define como explicativo, debido a que está orientado a describir cómo influye la motivación en la productividad de los trabajadores de la Gerencia de Infraestructura de Telefónica del Perú. Se aplicaron las técnicas de encuestas con preguntas estructuradas en un cuestionario ad-hoc y entrevistas a profundidad para revisar las estrategias aplicadas; las primeras a los trabajadores de línea y las entrevistas a los jefes o líderes del área.

La población de la investigación está compuesta por 33 trabajadores de las tres jefaturas de la Gerencia de Infraestructura. La muestra tomada fue del tipo discrecional, es decir, bajo el criterio del investigador; se consideró como muestra al $30 \%$ de la población.

\section{MARCO TEÓRICO}

\section{Motivación}

El deseo de conocer, predecir o influir la conducta laboral de los individuos ha llevado a muchos científicos a estudiar las causas y consecuencias de la motivación y satisfacción en el trabajo, estudios que se han realizado bajo diferentes enfoques en el intento de explicar los factores que determinan las actitudes de los trabajadores. Para la Escuela Clásica de la Administración, la motivación laboral era un problema de fácil resolución porque suponían que un hombre racional estaba orientado por el deseo de escapar al hambre y de aumentar sus ganancias, por lo que se conseguiría un mecanismo motivacional altamente eficiente si se lograba establecer un medio que conectara las ganancias con el rendimiento. La Escuela de Relaciones Humanas introduce mayores distinciones en este tema, al comprender la complejidad del ser humano, su sociabilidad y la amplitud de sus necesidades.

Según lo anteriormente dicho, el problema de la motivación no es solo el incentivar a las personas que pertenecen al sistema, aceptando sus condiciones. Si éste fuera el único problema, se correría el riesgo de contar con un personal poco interesado en el trabajo y que hace lo mínimo para no ser despedido. Como los resultados no son muy satisfactorios, hay que motivar adicionalmente a las personas 
que ya han ingresado, para que cumplan en la mejor manera posible, en cantidad y calidad, su rol. La motivación humana se define como «...un estado emocional que se genera en una persona como consecuencia de la influencia que ejercen determinados motivos». Así mismo, la motivación laboral puede ser definida como «...las fuerzas que actúan sobre el trabajador y originan que se comporte de una manera determinada, dirigida hacia las metas, condicionados por la capacidad del esfuerzo de satisfacer alguna necesidad individual».

Debido a que los motivos para desarrollar su trabajo por parte de los empleados influyen en la productividad, se constituye en una de las tareas de los gerentes encaminar efectivamente la motivación del empleado hacia el logro de las metas de la organización.

\section{Teorías}

A continuación se presentan algunas teorías conocidas de motivación que intentan explicar la conducta humana, tratando de identificar qué factores los motiva:

a) El modelo de la jerarquía de Maslow.

En la Pirámide de Maslow, el autor propuso una estratificación de las necesidades en cinco niveles jerarquizados de forma que el individuo debe de satisfacer cada nivel.

El primer nivel corresponde a las necesidades fisiológicas (comer, beber, dormir; laboralmente, el salario y condiciones laborales mínimas). El segundo nivel corresponde a la necesidad de protección y seguridad, en el aspecto laboral equivale a las condiciones de seguridad laborales, estabilidad en el empleo, seguridad social y salario superior al mínimo. El tercer nivel se refiere a las necesidades sociales como vinculación, cariño, amistad, etcétera, y en el ámbito del trabajo son las posibilidades de interactuar con otras personas, el compañerismo, relaciones laborales. El cuarto nivel corresponde a las necesidades de consideración y mantenimiento de un estatus que en el ámbito del empleo sería el poder realizar tareas que permitan un sentimiento de logro y responsabilidad, recompensas, promociones y reconocimiento. El nivel más alto implica la autorrealización personal que laboralmente se refiere a la posibilidad de utilizar plenamente las habilidades, capacidades y creatividad. b) La teoría ERG de Alderfer

Clayton Alderfer propone ciertos cambios a la Pirámide de Maslow, referidos a los niveles de necesidades de las personas, que deben tenerse en cuenta en el ámbito de la motivación. El mismo considera que los trabajadores deben de cubrir:

- Necesidades de Existencia (E), que incluyen junto a las necesidades fisiológicas y de seguridad de Maslow las condiciones de trabajo y las retribuciones.

- Necesidades de Relación (R), agrupan las necesidades sociales y de consideración de Maslow. Según Alderfer, estas necesidades se satisfacen más con una interacción abierta, correcta y honesta que por mantener relaciones agradables eludiendo la crítica.

- Necesidades de Desarrollo (D), que se refieren al deseo de autoestima y autorrealización a través de una fuerte implicación en la dinámica laboral y por la completa utilización de las habilidades, capacidades y creatividad.

c) La teoría de las necesidades aprendidas de McClelland.

McClelland considera que los impulsos motivacionales están en relación con necesidades de logro, afiliación y poder. Las características que definen a los que se orientan hacia el logro son la búsqueda de responsabilidad personal y la superación de retos a fin de alcanzar metas alcanzables. El logro es importante en sí mismo y no por las recompensas que lo acompañen. En el ámbito laboral, las personas motivadas por el logro siempre quieren mejorar todo en su medio ambiente, tratan de encontrar maneras más eficaces para hacer una tarea, de dominar su actividad desarrollando habilidades e innovaciones para la solución de problemas.

El autor también afirma que, además del motivo de logro, las necesidades de poder y afiliación son importantes pero deben de estar subordinadas a las de logro. Las personas con alta necesidad de poder desean poseerlo en su trabajo, e influir en las personas y en las situaciones, para lo que están dispuestos a correr riesgos. Suelen realizar acciones que afectan a la conducta de otros y despiertan en ellas fuertes emociones. 
La teoría de McClelland se adapta a la investigación porque los individuos muchas veces no necesitan factores que los inclinen a estar motivados en sus acciones, simplemente sienten una motivación por lograr algo y su motivación se centra únicamente en alcanzarlo.

d) La teoría de la motivación - higiene de Herzberg.

Existe una relación entre los factores intrínsecos y la satisfacción laboral, y entre los factores extrínsecos y la insatisfacción. Algunos factores intrínsecos o motivadores son: la realización, el reconocimiento, el trabajo mismo, la responsabilidad, el progreso y el desarrollo. Estos aspectos están relacionados con la satisfacción. Los factores extrínsecos o higiénicos, como el salario, la administración, la supervisión, las relaciones interpersonales, las políticas y la estructura administrativa de la compañía y las condiciones laborales si están presentes no originan motivación, pero evitan la insatisfacción.

Herzberg investigó la pregunta ¿qué quieren las personas de su trabajo? De las respuestas obtenidas acumuló información sobre diversos factores que afectaban los sentimientos de los trabajadores sobre sus empleos. Surgieron dos tipos de factores:

- Factores motivadores. Que incluyen el trabajo en sí mismo, el reconocimiento, la responsabilidad y los ascensos. Todos ellos se relacionan con los sentimientos positivos de los empleados acerca de su trabajo, los que a su vez se relacionan con las experiencias de logros, reconocimiento y responsabilidad del individuo.

Llevando esto al ámbito laboral, serían trabajo estimulante, sentimiento de autorrealización, reconocimiento de una labor bien realizada, cumplimiento de metas, y objetivos.

- Factores de higiene. Incluyen las políticas de administración de la organización, la supervisión técnica, el sueldo o salario, las prestaciones, las condiciones de trabajo y las relaciones interpersonales. Todos estos se relacionan con los sentimientos negativos de las personas hacia su trabajo y con el ambiente en el cual éste se realiza.

Llevando esto al ámbito laboral, serían factores económicos (sueldos, salarios, prestaciones sociales), condiciones laborales (entorno físico seguro), seguridad (privilegios de antiguiedad), factores sociales (clima organizacional, relaciones personales).

En conclusión, los motivadores son factores intrínsecos, vinculados directamente con la satisfacción en el trabajo y que pertenecen en gran parte al mundo interno de la persona.

Los factores de higiene son extrínsecos, es decir externos al trabajo, actúan como recompensas a causa del alto desempeño si la organización lo reconoce. Cuando son adecuados en el trabajo, calman a los empleados haciendo así que no estén insatisfechos y se desenvuelvan en un adecuado ambiente de trabajo.

Adicionalmente se consideran algunas investigaciones realizadas sobre la motivación.

En la tesis "Análisis de la Motivación en el personal Administrativo de la Empresa F.M.F. Construcciones C.A." de Ortega (2005) se concluye que los empleados de la organización consideran la motivación como un factor importante para la realización de sus labores cotidianas. La falta de políticas de incentivos influye negativamente en la prestación de servicios; la mayoría de los empleados no asocian un alto rendimiento con reconocimientos por parte de la empresa; el personal no se siente satisfecho con la remuneración percibida y que los empleados no se encuentran motivados al trabajo por parte de la empresa.

Recomienda que la empresa ofrezca capacitaciones sobre relaciones personales, comunicación y motivación para mejorar la calidad del medio ambiente en el cual se desenvuelven. Que cultive la cultura del reconocimiento, que se identifique las necesidades de los empleados y que se les escuche.

En la tesis "La motivación laboral y su incidencia en el desempeño organizacional: un estudio de caso" de Delgado (2010) se concluye que no hay un proceso de inducción donde se transmitan la visión, misión, valores de la empresa que son aspectos importantes que contribuyen a preparar y formar al talento humano, además de establecer un vínculo entre la filosofía de gestión de la empresa y los empleados.

Recomienda delimitar previamente los factores motivadores a tomar en cuenta, enfocarse en la 
satisfacción y crecimiento personal y profesional de todos sus empleados, independientemente del cargo que ocupan, y que se tenga en mente siempre una visión a futuro del negocio.

En la tesis "Motivación laboral" de Gonzales Esquivel (2002) se concluye que los jefes deben conocer bien a sus trabajadores, y el medio social del cual son parte integrante y que hay mejores maneras de motivar al trabajador, tales como elevar la autoestima de la persona, fomentar su iniciativa para ser creativo y productivo, que la motivación constituye un factor muy importante para llevar a cabo cualquier actividad, y que particularmente es necesario motivar al trabajador mejorando su economía.

Recomienda que los jefes deben hacer uso de técnicas adecuadas que estén de acuerdo a los intereses y necesidades de los trabajadores ya que con esto se puede lograr una motivación dirigida hacia el alcance de metas definidas.

En la tesis "El Impacto de la Motivación y el Liderazgo con el Rendimiento Laboral en una empresa de servicios de Lima Metropolitana" de Vargas Del Pino (2010) se concluye que la motivación de logro, la motivación de afiliación, la motivación de poder y el liderazgo se relacionan positiva y significativamente con el rendimiento laboral.

Se sugiere evaluar periódica y sistemáticamente las variables de motivación de logro, de afiliación, de poder y liderazgo dada su relación, debidamente comprobada como elementos incentivadores del rendimiento laboral; se recomienda ofrecer charlas y talleres de motivación laboral al personal directivo, administrativo, técnico y obrero, en especial a los supervisores directos. Recomienda también implantar un plan de reconocimientos, revisar las tareas que llevan a cabo los trabajadores y brindar más confianza al grupo, desarrollar líneas de investigación orientadas a asegurar y mejorar la puesta en práctica de programas de capacitación innovadores en materia laboral, e identificar personas con cualidades de liderazgo para ocupar diferentes responsabilidades o ser reserva de futuros cuadros.

En la tesis "Relación de los esquemas de remuneración con la productividad laboral de los empleados: empresa SUMMMA S.A." de Rodríguez Guerra (2008) se concluye que hay una estrecha relación entre los esquemas de remuneración y la productividad laboral con el volumen de producción, el uso de insumos y la calidad de la produc- ción de bienes o servicios, que son apreciados por los trabajadores como sistemas que permiten elevar y/o mejorar los niveles de productividad.

Recomienda que la empresa conozca qué se espera de los trabajadores en relación a los volúmenes de producción, cualidades y características del producto o servicio que hace, y sobre el aprovechamiento de insumos para que la empresa incorpore estos criterios a los esquemas de remuneración y hacerlos del conocimiento del personal.

\section{RENDIMIENTO LABORAL}

El rendimiento es una proporción entre el resultado obtenido y los medios que se utilizaron. En el ámbito de las empresas, la noción de rendimiento refiere al resultado que se obtiene por cada unidad que realiza una actividad, ya sea un departamento, una oficina o un único individuo. Cabe destacar que el concepto de rendimiento se encuentra vinculado al de efectividad o de eficiencia. La efectividad mide la capacidad de alcanzar un efecto deseado. La eficiencia, por su parte, hace referencia a la capacidad de alcanzar dicho efecto con la menor cantidad de recursos posibles.

En el campo del rendimiento laboral es necesario precisar que la productividad es considerada como el rendimiento, en calidad y cantidad, producido por un trabajador en determinada unidad de tiempo.

Modernamente se considera que la variable "productividad" es un tipo de efectividad organizacional. Por lo tanto, el desempeño de la gente en las organizaciones puede considerarse como el reflejo del logro de importantes objetivos de la organización y la predicción de la productividad del trabajador así como de los factores que contribuyen de manera decisiva para lograr la comprensión del comportamiento humano.

Se ha establecido que la productividad es el resultado de la intervención de un vasto número de factores, tanto individuales (el trabajador) como situacionales (naturaleza de la tarea, la empresa); tanto subjetivos (clima laboral, motivaciones, etcétera) como objetivos (condiciones materiales, salarios, etcétera); tanto racionales, como irracionales. Entre los principales factores individuales pueden considerase los factores físicos del trabajador (coordinación motora, destreza, fuerza, resistencia, limitaciones físicas), factores de entrenamiento (instrucción, capacitación, experiencia, adiestramiento, etcétera), factores 
psicológicos (agudeza sensorial, nivel intelectual, capacidad de aprendizaje, aptitudes, habilidades, personalidad, motivación, satisfacción laboral, moral, etcétera). Entre los principales factores situacionales pueden considerarse los relacionados con la naturaleza de la tarea (mayor o menor complejidad, entrenamiento, fatiga, tedio, descanso, iluminación, ventilación, ruidos) y los relacionados con la empresa (tipo de supervisión, incentivos, remuneración, promociones, sueldos, etcétera).

En la actualidad, los especialistas centran su atención en tres aspectos que consideran los más importantes para el rendimiento laboral: habilidades perceptuales y motoras, conducta de procesamiento de la información y conducta de atención o vigilancia.

\section{ANÁLISIS DE LA INFORMACIÓN}

Para la presente investigación se utilizó como técnica de recolección de datos un cuestionario aplicado a los trabajadores de la Gerencia de Infraestructura de Telefónica del Perú, como herramienta para evaluar la motivación, y una entrevista de profundidad a los jefes correspondientes.

A continuación se presentan los resultados.

Para la recolección de datos se realizaron encuestas cuyos resultados presentamos a continuación.

\section{1. ¿Recibe usted algún tipo de motivación por parte de sus superiores?}

\begin{tabular}{|l|r|r|r|r|}
\hline Recibe Motivación & \multicolumn{1}{|c|}{ fi } & \multicolumn{1}{c|}{ hi } & \multicolumn{1}{c|}{ Fi } & \multicolumn{1}{c|}{ Hi } \\
\hline SI & 5 & $50 \%$ & 5 & $50 \%$ \\
\hline No & 5 & $50 \%$ & 10 & $10 \%$ \\
\hline $\mathrm{N}=$ & 10 & & & \\
\hline
\end{tabular}

El 50\% de los trabajadores encuestados manifestaron que no reciben ningún tipo de motivación y el otro $50 \%$ señaló que si recibe motivación por parte de sus superiores.

\section{2. ¿Qué forma de motivación prefiere o preferiría?}

\begin{tabular}{|l|r|r|r|r|}
\hline Tipo de motivación & \multicolumn{1}{|c|}{$\mathrm{fi}$} & \multicolumn{1}{c|}{ hi } & \multicolumn{1}{c|}{$\mathrm{Fi}$} & \multicolumn{1}{l|}{$\mathrm{Hi}$} \\
\hline Emotiva & 5 & $50 \%$ & 5 & $50 \%$ \\
\hline Material & 5 & $50 \%$ & 10 & $10 \%$ \\
\hline $\mathrm{N}=$ & 10 & & & \\
\hline
\end{tabular}

El 50\% de los trabajadores encuestados manifestaron que prefieren una motivación material (extrínseca) y el otro $50 \%$ señaló que prefiere la emotiva (intrínseca).

3. Si la motivación que se le dieran fuera material, ¿qué preferiría recibir?

\begin{tabular}{|l|r|r|r|r|}
\hline Motivación material & \multicolumn{1}{|c|}{ fi } & \multicolumn{1}{c|}{ hi } & \multicolumn{1}{c|}{ Fi } & \multicolumn{1}{c}{ Hi } \\
\hline Bonos & 10 & $100 \%$ & 10 & $100 \%$ \\
\hline Regalos & 0 & $0 \%$ & 10 & $100 \%$ \\
\hline Viajes & 0 & $0 \%$ & 10 & $100 \%$ \\
\hline Otros & 0 & $0 \%$ & 10 & $100 \%$ \\
\hline $\mathrm{N}=$ & 10 & & & \\
\hline
\end{tabular}

El 100\% de los trabajadores encuestados manifestaron que de recibir una motivación extrínseca preferirían que sean bonos a su salario.

4. Si la motivación que le dieran fuera emotiva, ¿qué preferiría recibir?

\begin{tabular}{|l|r|r|r|r|}
\hline Motivación emotiva & \multicolumn{1}{|c|}{$\mathrm{fi}$} & \multicolumn{1}{c|}{ hi } & \multicolumn{1}{c|}{$\mathrm{Fi}$} & \multicolumn{1}{c}{$\mathrm{Hi}$} \\
\hline Reconocimientos & 4 & $40 \%$ & 4 & $40 \%$ \\
\hline Felicitaciones & 3 & $30 \%$ & 7 & $70 \%$ \\
\hline Otros & 3 & $30 \%$ & 10 & $100 \%$ \\
\hline $\mathrm{N}=$ & 10 & & & \\
\hline
\end{tabular}

El $40 \%$ de los trabajadores encuestados manifestaron que de recibir una motivación intrínseca preferirían que sean reconocimientos delante de sus compañeros, mientras que el $30 \%$ de ellos manifestaron preferir las felicitaciones personales.

\section{Grado en que se encuentra satisfecho con su ambiente laboral}

\begin{tabular}{l|r|r|r|r}
\hline Satisfacción del ambiente & \multicolumn{1}{|c|}{$\mathrm{fi}$} & \multicolumn{1}{c|}{ hi } & \multicolumn{1}{c|}{$\mathrm{Fi}$} & \multicolumn{1}{c}{$\mathrm{Hi}$} \\
\hline Muy satisfecho & 1 & $10 \%$ & 1 & $10 \%$ \\
\hline Satisfecho & 7 & $70 \%$ & 8 & $80 \%$ \\
\hline Me es indiferente & 1 & $10 \%$ & 9 & $90 \%$ \\
\hline Insatisfecho & 1 & $10 \%$ & 10 & $100 \%$ \\
\hline Muy insatisfecho & 0 & $0 \%$ & 10 & $100 \%$ \\
\hline $\mathrm{N}=$ & 10 & & & \\
\hline
\end{tabular}

El 70\% de los trabajadores encuestados manifestaron encontrarse satisfechos con su ambiente de trabajo.

\section{6. ¿Cómo calificaría la relación con su superior?}

\begin{tabular}{|l|r|r|r|r|}
\hline Relación con su superior & \multicolumn{1}{|c|}{$\mathrm{fi}$} & \multicolumn{1}{|c|}{ hi } & \multicolumn{1}{c|}{$\mathrm{Fi}$} & \multicolumn{1}{l|}{$\mathrm{Hi}$} \\
\hline Muy buena & 5 & $50 \%$ & 5 & $50 \%$ \\
\hline Buena & 3 & $30 \%$ & 8 & $80 \%$ \\
\hline Ni buena ni mala & 2 & $20 \%$ & 10 & $100 \%$ \\
\hline Mala & 0 & $0 \%$ & 10 & $100 \%$ \\
\hline Muy mala & 0 & $0 \%$ & 10 & $100 \%$ \\
\hline $\mathrm{N}=$ & 10 & & & \\
\hline
\end{tabular}


El 50\% de los trabajadores encuestados manifestaron tener una muy buena relación con sus superiores, mientras que ninguno manifestó tener una relación mala o muy mala con ellos.

7. ¿Con qué frecuencia recibe un buen trato de sus superiores?

\begin{tabular}{|l|r|r|r|r|}
\hline Buen trato de sus superiores & \multicolumn{1}{c|}{$\mathrm{fi}$} & \multicolumn{1}{c|}{ hi } & \multicolumn{1}{c|}{$\mathrm{Fi}$} & \multicolumn{1}{c}{$\mathrm{Hi}$} \\
\hline Siempre & 5 & $50 \%$ & 5 & $50 \%$ \\
\hline Casi siempre & 2 & $20 \%$ & 7 & $70 \%$ \\
\hline Regularmente & 1 & $10 \%$ & 8 & $80 \%$ \\
\hline Casi nunca & 2 & $20 \%$ & 10 & $100 \%$ \\
\hline Nunca & 0 & $0 \%$ & 10 & $100 \%$ \\
\hline $\mathrm{N}=$ & 10 & & & \\
\hline
\end{tabular}

El 50\% de los trabajadores encuestados manifestaron recibir siempre un buen trato por parte de sus superiores mientras que ninguno manifestó no recibir nunca un buen trato por parte de ellos.

8. ¿Considera que puede dar su opinión libremente a cualquiera de sus superiores?

\begin{tabular}{|l|r|r|r|r|}
\hline Opinión libremente & \multicolumn{1}{c|}{$\mathrm{fi}$} & \multicolumn{1}{c|}{ hi } & \multicolumn{1}{c|}{$\mathrm{Fi}$} & \multicolumn{1}{l}{$\mathrm{Hi}$} \\
\hline Si & 7 & $70 \%$ & 7 & $70 \%$ \\
\hline No & 3 & $30 \%$ & 10 & $100 \%$ \\
\hline $\mathrm{N}=$ & 10 & & & \\
\hline
\end{tabular}

El 70\% de los trabajadores encuestados manifestaron poder dar su opinión libremente a sus superiores mientras que solo el $30 \%$ de ellos manifestaron no poder dar su opinión.

9. ¿Considera que sus opiniones son tomadas en cuenta?

\begin{tabular}{|l|r|r|r|r|}
\hline Opinión libremente & \multicolumn{1}{|c|}{$\mathrm{fi}$} & \multicolumn{1}{c|}{ hi } & \multicolumn{1}{c|}{$\mathrm{Fi}$} & \multicolumn{1}{c|}{$\mathrm{Hi}$} \\
\hline $\mathrm{Si}$ & 5 & $50 \%$ & 5 & $50 \%$ \\
\hline No & 1 & $10 \%$ & 6 & $60 \%$ \\
\hline A veces & 4 & $40 \%$ & 4 & $100 \%$ \\
\hline $\mathrm{N}=$ & 10 & & & \\
\hline
\end{tabular}

El 50\% de los trabajadores encuestados manifestaron que sus opiniones sí son tomadas en cuenta por sus superiores, mientras que el $40 \%$ de ellos manifestaron que no se toman en cuenta.

10.¿Cree usted que la motivación influye en su desempeño laboral?

\begin{tabular}{|l|r|r|r|r}
\hline Influencia de la motivación & \multicolumn{1}{c|}{ fi } & \multicolumn{1}{c|}{ hi } & \multicolumn{1}{c|}{$\mathrm{Fi}$} & \multicolumn{1}{c}{ Hi } \\
\hline $\mathrm{Si}$ & 9 & $90 \%$ & 9 & $90 \%$ \\
\hline No & 1 & $10 \%$ & 10 & $100 \%$ \\
\hline $\mathrm{N}=$ & 10 & & & \\
\hline
\end{tabular}

El 90\% de los trabajadores encuestados manifestaron que la motivación sí influye en su desempeño laboral, mientras que solo el 10\% manifestó que la motivación no influye en nada en el desempeño laboral.

Además de las encuestas que se les realizó a los colaboradores de las áreas, se hicieron también entrevistas a los jefes para ver desde su perspectiva la influencia de la motivación en la productividad de la empresa.

\section{Comprobación de las hipótesis según la encuesta}

HE1: Se afirma que al menos el $75 \%$ de los trabajadores prefieren recibir una motivación extrínseca. ¿Será cierta la afirmación?

\section{Hipótesis posibles}

Ho: El número de trabajadores que manifestaron preferir una motivación extrínseca es igual o mayor al 75\% del total de encuestados.

H1: El número de trabajadores que manifestaron preferir una motivación extrínseca es menor al 75\% del total de encuestados.

\section{a) Regiones}

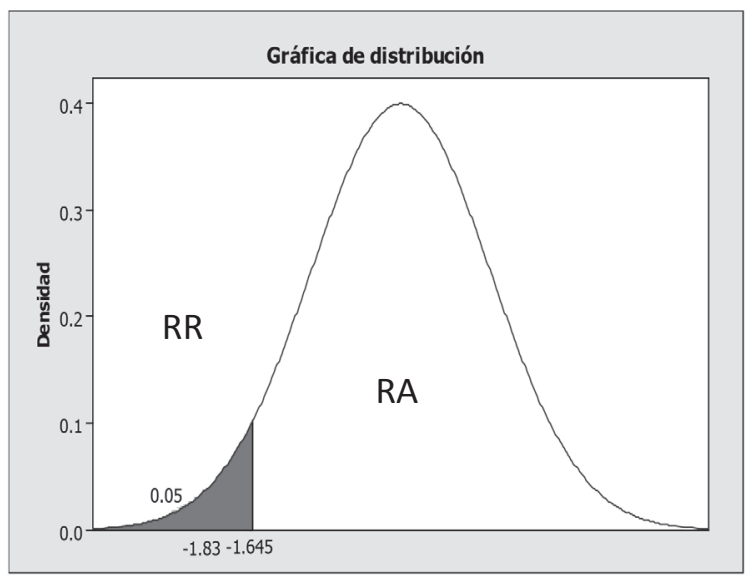

$$
\begin{array}{|c|c|c|c|c|}
\hline \mathrm{X} & \mathrm{n} & \text { Muestra } \mathrm{p} & \text { Valor } \mathrm{Z} & \text { Valor } \mathrm{P} \\
\hline 5 & 10 & 0.50 & -1.83 & 0.034 \\
\hline \multicolumn{5}{c}{\text { P- valor }<\propto \rightarrow \text { Ho se rechaza }} \\
0.034<0.05 \\
\text { Zo } \in \text { RR } \rightarrow \text { Ho se rechaza }
\end{array}
$$

La afirmación se rechaza; es decir, menos del $75 \%$ de los trabajadores encuestados manifestaron preferir la motivación extrínseca.

HE2: Se afirma que al menos el $80 \%$ de los trabajadores manifestaron que la motivación SI influye en su desempeño laboral. ¿Será cierta la afirmación? 


\section{Hipótesis posibles}

Ho: El número de trabajadores que manifestaron que la motivación SI influye en su desempeño laboral es igual o mayor al $80 \%$ del total de encuestados.

H1: El número de trabajadores que manifestaron que la motivación SI influye en su desempeño laboral es menor al $80 \%$ del total de encuestados.

\section{a) Regiones}

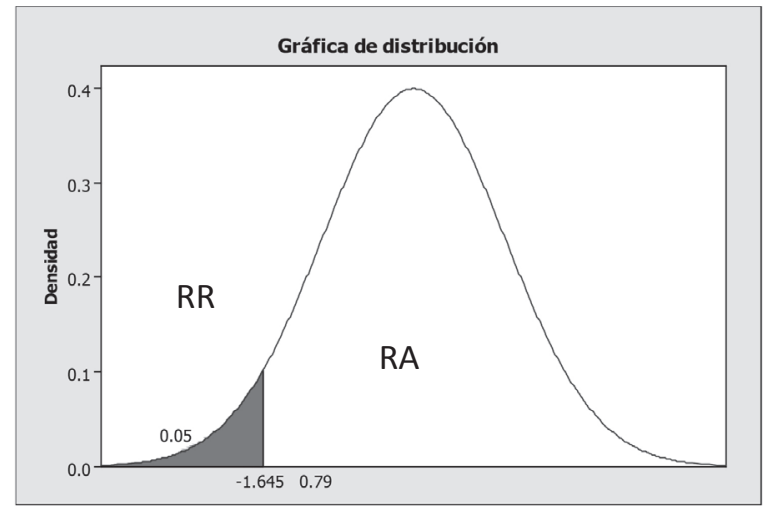

\begin{tabular}{|c|c|c|c|c|}
\hline $\mathrm{X}$ & $\mathrm{n}$ & Muestra $\mathrm{p}$ & Valor $\mathrm{Z}$ & Valor $\mathrm{P}$ \\
\hline 9 & 10 & 0.90 & 0.79 & 0.893 \\
\hline
\end{tabular}

P- valor $<\propto \rightarrow$ Ho se acepta

$0.89<0.05$

$\mathrm{Zo} \in \mathrm{RA} \rightarrow$ Ho se acepta

La afirmación se acepta; es decir, al menos el $80 \%$ de los trabajadores encuestados manifestaron que la motivación SI influye en el desempeño laboral.

Considerando los resultados de las encuestas y las entrevistas realizadas a los funcionarios del área de Infraestructura de Telefónica del Perú, se comprueban las hipótesis de estudio:

- "El ejercicio de una buena motivación de parte de los ejecutivos hacia los colaboradores contribuye a un mejor resultado en la productividad del área de Infraestructura de Telefónica del Perú".

Se comprueba que la motivación es uno de los factores determinantes, para alcanzar los objetivos, mostrados a través del cumplimiento de indicadores de gestión; también es esencial para generar un buen clima laboral.

- "Los factores motivacionales extrínsecos, son los que generan una mayor satisfacción a los empleados." (primera hipótesis especifica).
El $50 \%$ de los encuestados manifiesta que lo considera importante pero el otro $50 \%$ considera a la motivación intrínseca más importante; además se observó que en el caso de la motivación extrínseca se prefiere las recompensas relacionadas con el dinero; sin embargo, este es un tema transversal a la empresa, sobre el cual los jefes del área no tienen autonomía.

- "Los trabajadores motivados tienen un mejor desempeño laboral.” (segunda hipótesis específica)

Se valida; tanto los colaboradores como los jefes coinciden en indicar que la motivación es importante para un buen desempeño que a su vez influye directamente en una mayor productividad para el área, logrando mayor satisfacción de los clientes.

\section{CONCLUSIONES}

Se muestran las siguientes conclusiones:

1. Se ha verificado en la investigación que uno de los aspectos importantes para lograr mayor productividad en la Gerencia de Infraestructura de Telefónica del Perú es la motivación; que su influencia para mejorar el desempeño y alcanzar los objetivos es fundamental.

2. Se ha identificado con igual valoración tanto la motivación extrínseca (recompensas materiales, dinero) como la motivación intrínseca (reconocimiento y la valoración de su desempeño laboral). En este aspecto, el carácter y las actitudes de los jefes ejercen una influencia referencial hacia los colaboradores.

3. Debido a que la motivación extrínseca, especialmente la dependiente del dinero, no puede ser gestionada particularmente por los jefes de la Gerencia de Infraestructura de Telefónica del Perú, porque es un aspecto manejado por la empresa en su conjunto, la motivación utilizada más frecuentemente por los jefes en el área es la motivación intrínseca y una de las estrategias utilizadas es la comunicación y el feedback.

4. Tanto los colaboradores como los jefes indican que la motivación es importante para su buen desempeño, sin embargo, es menester continuar permanentemente buscando formas nuevas de motivar al personal para mantenerlo con buen ánimo en la lucha por alcanzar los objetivos. 


\section{RECOMENDACIONES}

En el caso específico de la productividad, una de las formas en la que se midió fue por medio de algunos indicadores que tienen los jefes de la Gerencia de Infraestructura de Telefónica del Perú, uno de ellos relacionado con el presupuesto que cada área tiene para desarrollar sus proyectos; es importante aquí buscar la participación de todos, que se sientan involucrados, que su opinión sea escuchada y tomada en cuenta.

Se recomienda a los jefes de área buscar mecanismos que le permitan conocer bien a sus colaboradores para identificar qué tipo de motivación aplicar y diseñar la estrategia particular para cada caso.

Si bien los jefes de la Gerencia de Infraestructura de Telefónica del Perú no tienen el control para proporcionar alguna recompensa de forma material (motivación extrínseca) a sus colaboradores, lo que si deben mantener y reforzar es el uso de la motivación intrínseca, pero no solo en la oficina si no también fuera de ésta para así buscar que los colaboradores se sientan más comprometidos con el equipo y más identificados con sus superiores.

Es muy importante tener en cuenta la perspectiva de los colaboradores, por lo que se recomienda a los jefes estar atentos a la opinión que de ellos tienen sus colaboradores.

\section{LITERATURA CITADA}

Delgado, María. (2010). "La motivación laboral y su incidencia en el desempeño organizacional: un estudio de caso". Tesis para obtener el grado de magíster. Universidad de Central de Venezuela.

Etkin, J. (2007). Capital Social y Valores en las Organizaciones Sustentables. Granica S.A., Buenos Aires.

Fernández, E. (2010). Administración de empresas un enfoque interdisciplinar. Paraninfo, Madrid.

Gonzales Esquivel, David Antonio. (2002). "Motivación laboral". Tesis para obtener el grado de magíster. Universidad Autónoma de Nuevo León, México.

Köhler, H.D., y Artiles, M. (2010). Manual de la sociología del trabajo y de las relaciones laborales. Delta Publicaciones, Madrid.

Martínez, M. (2013). La gestión empresarial. Ediciones Díaz de Santos S.A., Madrid.

Patricio, D. (2011). Manual de recursos humanos. Esic Editorial, Madrid.

Puchol, L. (2007). Dirección y gestión de recursos humanos. Madrid. Ediciones Díaz de Santos S.A.

Quintanilla, I., Bonavía, T. (2005). Psicología y Economía. Valencia. Maite Simon.

Robbins, S. (2004). Comportamiento Organizacional. México. Pearson Educación.

Robbins, S., Coulter, M (2006). Administración. México. Prentice Hall.

Rodríguez Guerra, Alejandro. (2008). "Relación de los esquemas de remuneración con la productividad laboral de los empleados: empresa SUMMMA SA.”. Universidad Autónoma de Aguascalientes, México. 